Published in final edited form as:

Clin Cancer Res. 2015 September 15; 21(18): 4055-4061. doi:10.1158/1078-0432.CCR-15-0304.

\title{
A phase I trial of the anti-KIR antibody IPH2101 and lenalidomide in patients with relapsed/refractory multiple myeloma
}

\author{
Don M. Benson Jr. ${ }^{1}$, Adam D. Cohen ${ }^{2}$, Sundar Jagannath ${ }^{3}$, Nikhil C. Munshi ${ }^{4}$, Gary \\ Spitzer $^{5}$, Craig C. Hofmeister ${ }^{1}$, Yvonne A. Efebera ${ }^{1}$, Pascale Andre ${ }^{6}$, Robert Zerbib ${ }^{6}$, and \\ Michael A. Caligiuri ${ }^{1}$ \\ ${ }^{1}$ The Ohio State University Comprehensive Cancer Center, Columbus, $\mathrm{OH}$ \\ ${ }^{2}$ University of Pennsylvania Abramson Cancer Center, Philadelphia, PA \\ ${ }^{3}$ Mount Sinai School of Medicine, New York, NY \\ ${ }^{4}$ Dana Farber Cancer Institute, Boston, MA \\ ${ }^{5}$ St. Francis Hospital, Greenville, SC \\ ${ }^{6}$ Innate Pharma, Marseille, France
}

\begin{abstract}
PURPOSE-Natural killer (NK) cells may play an important role in the immune response to multiple myeloma (MM); however, MM cells express killer immunoglobulin-like receptor (KIR) ligands to prevent NK cell cytotoxicity. Lenalidomide can expand and activate NK cells in parallel with its direct effects against MM; however, dexamethasone may impair these favorable immunomodulatory properties. IPH2101, a first-in-class anti-inhibitory KIR antibody, has acceptable safety and tolerability in MM as a single agent. The present work sought to characterize lenalidomide and IPH2101 as a novel, steroid-sparing, dual immune therapy for MM.
\end{abstract}

EXPERIMENTAL DESIGN-A phase I trial enrolled 15 patients in three cohorts. Lenalidomide was administered per os at 10mg on cohort 1 and $25 \mathrm{mg}$ on cohorts 2 and 3 days $1-21$ on a 28 -day cycle with IPH2101 given intravenously on day 1 of each cycle at $0.2 \mathrm{mg} / \mathrm{kg}$ on cohort $1,1 \mathrm{mg} / \mathrm{kg}$ on cohort 2 , and $2 \mathrm{mg} / \mathrm{kg}$ on cohort 3 . No corticosteroids were utilized. The primary endpoint was safety, secondary endpoints included clinical activity, pharmacokinetics (PK) and pharmacodynamics (PD).

RESULTS-The biologic endpoint of full KIR occupancy was achieved across the IPH2101 dosing interval. PD and PK of IPH2101 with lenalidomide were similar to data from a prior single agent IPH2101 trial. Five serious adverse events (SAEs) were reported. Five objective responses occurred. No autoimmunity was seen.

Corresponding Author: Don M Benson Jr, MD PhD, Division of Hematology, B310 Starling Loving Hall, The Ohio State University Comprehensive Cancer Center, $320 \mathrm{~W} 10^{\text {th }}$ Ave Columbus OH USA 43210-1240, Phone: 614.293.8605, Fax: 614.293.7526, Don.benson@osumc.edu.

Disclosure of Conflicts of Interest: No other authors have conflicts to disclose.

Authorship roles: DMB designed research, enrolled subjects, analyzed and interpreted data and wrote the manuscript. ADC, SJ, NM, GS, CCH, YAE participated in data acquisition, anaylsis and interpretation. PA and RZ provided reagents, and analyzed and interpreted data. MAC designed research, analyzed and interpreted data. All authors approved the final version of the manuscript. 
CONCLUSIONS-These findings suggest that lenalidomide in combination with anti-inhibitory KIR therapy warrants further investigation in MM as a steroid-sparing, dual immune therapy. This trial was registered at www.clinicaltrials.gov (reference: NCT01217203).

\section{Keywords}

Multiple myeloma; natural killer cells; anti-KIR therapy; lenalidomide

\section{INTRODUCTION}

Novel therapies including immunomodulating agents (e.g., thalidomide, lenalidomide, pomalidomide) and proteasome inhibitors (bortezomib, carfilzomib) have significantly improved patient outcomes with multiple myeloma (MM)(1). Immunomodulatory agents such as lenalidomide may exert anti-MM efficacy, in part, through expansion and activation of natural killer (NK) cells which have been shown to play an important role in the immune response against MM (2-9). However, MM cells utilize specific immunoevasive strategies to reduce NK cell recognition and cytotoxicity (10-14).

Presently, lenalidomide is administered in combination with dexamethasone which may attenuate its favorable immunomodulatory properties $(15,16)$. Corticosteroids are the backbone of virtually every effective therapy for MM, yet these agents also confer substantial risk of toxicities (e.g., hypertension, glucose intolerance, osteoporosis, and psychiatric effects in addition to immune suppression). A prior study of lenalidomide plus high-dose dexamethasone (40 mg PO days 1-4, 9-12, and 17-20 on a 28-day cycle) versus lenalidomide plus low-dose dexamethasone (40 mg PO days 1, 8, 15, and 22 on a 28-day cycle) in newly diagnosed MM showed that although response rates were higher with highdose dexamethasone, overall survival was superior and less toxicity was observed with lowdose dexamethasone (17). An effective lenalidomide combination therapy, devoid of corticosteroids, would represent a significant advance in the treatment options for MM (18).

IPH2101 (formerly 1-7F9) is a first-in-class, humanized $\mathrm{IgG}_{4}$ monoclonal antibody against common inhibitory killer immunoglobulin-like receptors (KIRs) which disrupts inhibitory KIR-ligand interaction to promote NK cell recognition and lysis of tumor cells seeking to recapitulate the effects of KIR-ligand mismatch that mediate NK cell alloreactivity in haploidentical allogeneic stem cell transplantation $(19,20)$. MM cells upregulate surface expression of HLA class I molecules (which serve as inhibitory KIR ligands) making this receptor-ligand axis a provocative target for NK-cell mediated therapeutics (5). A singleagent, dose-escalation, phase I trial of IPH2101 in relapsed/refractory MM reached the biologic endpoint of full KIR blockade over dosing interval, with correlative evidence of NK cell activation and enhanced function, but without dose-limiting toxicity (21). In that trial, stable disease was observed in $34 \%$ of treated patients (21). Preclinical data suggest that the combination of lenalidomide and IPH2101 confer anti-MM effects through complementary mechanisms modulating the NK cell versus MM effect (22). With lenalidomide to augment NK cell function and IPH2101 to release NK cells from inhibition, the present phase I dose-escalation trial of this combination was conducted in patients with relapsed/refractory MM. No corticosteroids were utilized. 


\section{MATERIALS AND METHODS}

\section{Study objectives}

The primary objective of the trial was to determine the safety and tolerability of IPH2101 in combination with lenalidomide by NCI CTC Version 4.0 of May, 2009. The secondary objectives were to evaluate: the anti-MM activity, the pharmacokinetics and pharmacodynamics of IPH2101 in combination with lenalidomide, and to confirm the absence of immunogenicity of IPH2101.

\section{Study population}

Adult patients (ages 18 - 80 years) with relapsed/refractory MM according to International Myeloma Working Group (IMWG) definition after one or two prior lines of treatment were eligible for inclusion with: measurable disease, Eastern Cooperative Oncology Group performance status of $0-2$, adequate renal (calculated creatinine clearance $\geq 60 \mathrm{ml} / \mathrm{min}$ ), hepatic (bilirubin < 1.5xinstitutional upper limit of normal, ALT / AST < $3 \times$ institutional upper limit of normal), and bone marrow function (absolute neutrophil count $>1 \times 10^{9} / 1$ and platelet $275 \times 10^{9} / 1$ for patients with $<50 \%$ bone marrow plasma cells and $\geq 30 \times 10^{9} / 1$ for patients with $>50 \%$ bone marrow plasma cells). Eligibility also included meeting satisfactory conditions outlined in the mandatory RevAssist ${ }^{\circledR}$ program for lenalidomide dispensing. Patients could have had prior lenalidomide but had to have achieved at least partial response for 6 months and not have discontinued lenalidomide previously due to toxicity or intolerance. Patients with history of autoimmune disease, HIV, chronic hepatitis, or history of allogeneic transplantation were excluded. Concomitant use of corticosteroids was prohibited. The study was approved by Institutional Review Boards before initiation and was conducted in accordance with the Declaration of Helsinki. All subjects provided informed consent prior to participation.

\section{Study design}

IPH2101 was administered intravenously on day 1 of a 28-day cycle over three cohorts of escalating doses: $0.2 \mathrm{mg} / \mathrm{kg}$ (with $10 \mathrm{mg}$ lenalidomide orally days 1-21), $0.2 \mathrm{mg} / \mathrm{kg}$ (with 25 $\mathrm{mg}$ lenalidomide), and $1 \mathrm{mg} / \mathrm{kg}$ (with $25 \mathrm{mg}$ lenalidomide). A standard $3+3$ trial design was utilized with dosing cohorts added sequentially following interim safety and tolerability data review.

Patients were to receive 4 cycles of IPH2101 and lenalidomide and were eligible for an additional 4 cycles pending safety, tolerability and evidence of clinical benefit. Thereafter, patients were maintained on single agent lenalidomide. Dose modifications of IPH2101 were not permitted. Dose modifications for lenalidomide-related grade 3 or 4 hematologic AEs followed standard dose adaptation rules. For other grade 4 adverse events related to lenalidomide, treatment was held and restarted at next lower dose following resolution of toxicity if trial continuation was considered in the patient's best interest by the treating physician.

Dose limiting toxicity (DLT) was defined as any of the following in cycle 1 of treatment: grade 4 neutropenia lasting $\geq 7$ days or grade 3 neutropenia with fever $>38^{\circ} \mathrm{C}$, grade 4 
thrombocytopenia for patients with baseline platelet count $\geq 75 \times 10^{9} / 1$ (or $<10 \times 10^{9} / 1$, or with bleeding, for patients with baseline $>50 \%$ bone marrow plasma cells and platelet count $\left.30-75 \times 10^{9} / 1\right)$. Patients experiencing a lenalidomide related hematologic DLT were able to continue on trial if considered in the patient's best interest by the treating physician and if patient qualified for next cycle of therapy with dose reduction (however, the event was still counted towards establishment of a maximally tolerated dose). Any grade $\geq 3$ nonhematologic toxicity possibly or probably related to the combination that did not resolve or decrease to grade 2 or less within 3 days was also considered a DLT.

\section{Response criteria}

Objective responses were assessed by uniform International Myeloma Working Group (IMWG) criteria and minor response by European Group for Blood and Marrow Transplant (EBMT) criteria. Time to event endpoints (time to progression, progression free survival and duration of response) were defined according to IMWG guidelines.

\section{Correlative studies}

Pharmacokinetics (PK) and pharmacodynamics (PD) were analyzed as described previously (21). PK and PD of IPH2101 were assessed in combination with lenalidomide and compared to historical data from a prior single agent IPH2101 trial to assess any effects of concomitant administration of lenalidomide on the PK and PD of IPH2101. Cytokine profiles and lymphocyte subsets were serially analyzed over the course of the trial as described previously (21).

\section{Statistical considerations}

Descriptive statistics were used to summarize continuous data and categorical data were summarized by number / percentage of events. Progression-free survival was calculated using the Kaplan-Meier method.

\section{RESULTS}

\section{Patient characteristics}

In total, 15 patients (10 male, 5 female; median age 60 years, range 39-76) were enrolled between February, 2011 and December, 2013 and received at least 1 dose of IPH2101 and lenalidomide. Cohorts 1 and 3 were expanded to $n=6$ patients resulting from potential dose limiting toxicities (DLTs) described in detail below. There was an average of 3.5 years (range 1.25 - 10.1) from diagnosis to enrollment on the trial. Ten patients had one prior line of therapy, 5 patients had two prior lines. Twelve had had prior high-dose therapy with autologous stem cell transplantation. Ten had received prior lenalidomide (prior exposure to lenalidomide was mean of $6.8 \pm 6.5$ months, and time from lenalidomide exposure to first dose of IPH2101 and lenalidomide on study was average of $2 \pm 1$ year). Patient demographics, disease and prior treatment characteristics are summarized in Table 1. 


\section{Treatment, safety and toxicity}

Eight patients (53\%) completed 4 planned cycles of therapy. 5 patients (33\%) completed 8 cycles of therapy of which, 4 went onto maintenance therapy. Four patients $(27 \%)$ received reduced doses of lenalidomide, mainly related to neutropenia. In total, 46 treatment emergent adverse events (AEs) of grade $3 / 4$ severity were observed in $n=8$ patients and considered "possibly" or "probably" related to IPH2101 and/or lenalidomide (summarized in Table 2). Of 5 serious AEs, 3 were "possibly" or "probably" related to IPH2101 and/or lenalidomide. No patients permanently discontinued treatment for safety related to IPH 2101.

Potential dose-limiting toxicities (DLTs) were experienced by one patient in cohort 1 and one patient in cohort 3 . These two events were similar in nature and timing, characterized by fever and cytokine release on Cycle 1, Day 1 following administration of IPH2101 and lenalidomide. Both patients subsequently developed grade 4 leucopenia and neutropenia which resolved without growth factor support or other intervention within 72 hours. Cohorts 1 and 3 were both expanded without recurrence of the potential DLT. Both patients remained on study and did not experience similar reactions during subsequent cycles of therapy; however, neither experienced an objective response. Both patients with this DLT demonstrated profound increases in pro-inflammatory cytokines (IFN- $\gamma$, IL-6, TNF- $\alpha$ and MIP-1 $\beta$ as shown in Figure 1) following infusion of IPH2101 as compared to other patients in the study. The trial was amended to include anti-pyretic and anti-histamine prophylaxis, and no further infusion-related reactions were observed.

After completion of the study, one patient developed therapy-related myelodysplasia. This patient had previously received lenalidomide / dexamethasone induction therapy followed by intravenous melphalan $200 \mathrm{mg} / \mathrm{m}^{2}$ with autologous stem cell transplantation before relapsing and entering cohort 1 . The patient received lenalidomide $10 \mathrm{mg} /$ day during 28 months while on study with treatment interruptions transiently for neutropenia.

\section{IPH2101 PK and PD are not affected by co-administration of lenalidomide}

As in a prior single-agent trial, the dose of IPH2101 correlated with serum concentration over time (Figure 2A). KIR occupancy was consistent across the 28-day dosing interval for most patients at the $1 \mathrm{mg} / \mathrm{kg}$ dose of IPH2101 (Figure 2B). The administered dose of IPH2101 also correlated with the observed proportion of KIR occupancy (Figure 2C). A direct comparison of IPH2101 serum concentrations as a function of dose (Figure 2D) suggests that co-administration of lenalidomide did not significantly affect the PK of IPH2101 in the present study in comparison with data obtained in a prior single agent trial (21).

\section{Immunomodulatory correlates of IPH2101 and lenalidomide}

No statistically significant changes were observed in proportions of lymphocyte subsets or expression of markers of activation on lymphocytes over the course of the trial (data not shown). 


\section{Evaluation of clinical efficacy}

Although safety and tolerability were the primary endpoints of the trial, patients were evaluated for best response to therapy. There were 5 objective responses (Table 3): 2 very good partial responses (VGPR) and 3 partial responses (PR); out of these, objective responses occurred in 3 patients who had received prior lenalidomide / dexamethasone. One patient achieved a minor response (MR) and 6 achieved stable disease. The median time to best response observed (VGPR, PR, or MR) was 2.8 cycles (range $0.9-4.8$, Figure 3A). The median duration of response was 24 months ( $95 \% \mathrm{CI}=2.5$ to not reached). The median progression free survival was also 24 months $(95 \% \mathrm{CI}=2.5$ to not reached, Figure $3 \mathrm{~B})$.

\section{DISCUSSION}

NK cells appear to play an important role in the immune response to MM; however the disease utilizes specific strategies to evade NK cell detection and cytotoxicity (23). Among these immunoevasive mechanisms, MM cells may increase expression of inhibitory KIR ligands as the disease progresses (5). KIR-ligand mismatch has been shown to be a potential determinant of outcome in allogeneic stem cell transplantation in MM as in other hematologic malignancies $(20,24)$. Based on these concepts, disrupting KIR-ligand interaction as a means to prevent inhibitory signaling in NK cells to augment the NK cell versus MM effect has been a topic of ongoing investigation. An initial, single-agent, doseescalation study showed acceptable safety and tolerability with the anti-inhibitory KIR antibody, IPH2101, in patients with MM achieving the biologic endpoint of full KIR occupancy over the dosing interval (21).

Lenalidomide exerts direct anti-MM effects but also appears to confer favorable immunomodulatory effects on NK cells (2-4). Although the addition of dexamethasone to lenalidomide appears to enhance direct anti-MM activity, dexamethasone also appears to suppress lenalidomide's favorable immunomodulatory mechanisms particularly on NK cell function $(15,16)$. Thus, using lenalidomide as a means to augment NK cell number and function and IPH2101 to prevent inhibitory signaling, the present trial may be considered as the first "dual, innate immunotherapy" for MM.

Overall, the combination of lenalidomide and IPH2101 was well tolerated. Infusion-related events observed on cohorts 1 and 3 were abrogated by pre-medication with anti-pyretic and anti-histamine therapy. The PK and PD of IPH2101 did not appear to be affected by coadministration of lenalidomide and the pre-specified biologic endpoint of full KIR occupancy over the 28-day dosing interval was achieved. Objective responses were observed in $33.3 \%$ of patients with and without prior lenalidomide exposure, and median PFS was 24 months. A prior study characterizing lenalidomide as a single agent in relapsed/ refractory MM demonstrated a relatively similar response rate (26\%) to that observed in the present trial; however, median PFS was only 4.9 months with lenalidomide alone (25). Prior trials of lenalidomide plus high-dose dexamethasone in the relapsed/refractory setting showed a $60-61 \%$ response rate with median PFS of 11.1 months $(26,27)$. It is important to note that the present study was small, and such comparisons to studies of lenalidomide alone or with dexamethasone are merely speculative in nature. 
Therapeutic monoclonal antibodies (mAbs) such as IPH2101 comprise just one dimension (28) of the promising, rapidly expanding field of immunotherapy for MM which also includes NK- and T-cellular therapies (29,30), vaccines (30) and immunomodulating agents, among others. Tumor-directed mAbs $(31,32)$ as well as effector cell-targeted mAbs $(21,22,33)$ provide an opportunity for increasing precision with which to modulate immunity against MM, as well. The optimal combinations as well as the most appropriate clinical setting (e.g., smoldering, induction, maintenance, relapse) remain active areas of ongoing inquiry. The present data inform this process by demonstrating that the combination of antiKIR blockade with IPH2101 as a release from inhibition with lenalidomide to augment NK cell function appears to be safe and tolerable with preliminary evidence of efficacy. These findings justify further research into combination immune therapies designed to complement one another in enhancing immunity against MM as a therapeutic strategy.

\section{Acknowledgements}

Supported by National Cancer Institute P01 CA095426 (DMB, MAC).

DMB has received research funding from Innate Pharma. PA and RZ are employees of Innate Pharma. MAC is a member of the Supervisory Board of Innate Pharma. The authors confirm that neither the submitted manuscript nor any similar manuscript, in whole or in part is under consideration, in press, or published elsewhere.

\section{References}

1. Kumar SK, Rajkumar SV, Dispenzieri A, Lacy MQ, Hayman SR, Buadi FK, et al. Improved survival in multiple myeloma and the impact of novel therapies. Blood. 2008; 111:2516-2520. [PubMed: 17975015]

2. Davies FE, Raje N, Hideshima T, Lentzsch S, Young G, Tai YT, et al. Thalidomide and immunomodulatory derivatives augment natural killer cell cytotoxicity in multiple myeloma. Blood. 2001; 98:210-216. [PubMed: 11418482]

3. Hayashi T, Hideshima T, Akiyama M, Podar K, Yasui H, Raje N, et al. Molecular mechanisms whereby immunomodulatory drugs activate natural killer cells: clinical application. Br J Haematol. 2005; 128:192-203. [PubMed: 15638853]

4. Zhu D, Corral LG, Fleming YW, Stein B. Immunomodulatory drugs Revlimid (lenalidomide) and CC-4047 induce apoptosis of both hematological and solid tumor cells through NK cell activation. Cancer Immunol Immunother. 2008; 57:1849-1859. [PubMed: 18392823]

5. Carbone E, Neri P, Mesuraca M, Fulciniti MT, Otsuki T, Pende D, et al. HLA class I, NKG2D, and natural cytotoxicity receptors regulate multiple myeloma cell recognition by natural killer cells. Blood. 2005; 105:251-258. [PubMed: 15328155]

6. Sawanobori M, Suzuki K, Nakagawa Y, Inoue Y, Utsuyama M, Hirokawa K. Natural killer cell frequency and serum cytokine levels in monoclonal gammopathies: correlation of bone marrow granular lymphocytes to prognosis. Acta Haematol. 1997; 98:150-154. [PubMed: 9352746]

7. Osterborg A, Nilsson B, Bjorkholm M, Holm G, Mellstedt H. Natural killer cell activity in monoclonal gammopathies: relation to disease activity. Eur J Haematol. 1990; 45:153. [PubMed: 1699786]

8. Gonzalez M, San Miguel JF, Gascon A, Moro MJ, Hernandez JM, Ortega F, et al. Increased expression of natural-killer-associated and activation antigens in multiple myeloma. Am J Hematol. 1992; 39:84-89. [PubMed: 1550111]

9. Garcia-Sanz R, Gonzalez M, Orfao A, Moro MJ, Hernandez JM, Borrego D, et al. Analysis of natural killer-associated antigens in peripheral blood and bone marrow of multiple myeloma patients and prognostic implications. Br J Haematol. 1996; 93:81-88. [PubMed: 8611480] 
10. Frassanito MA, Silvestris F, Cafforio P, Silvestris N, Dammacco F. IgG M-components in active myeloma patients induce a down-regulation of natural killer cell activity. Int J Clin Lab Res. 1997; 27:48-54. [PubMed: 9144027]

11. Sawanobori M, Suzuki K, Nakagawa Y, Inoue Y, Utsuyama M, Hirokawa K. Natural killer cell frequency and serum cytokine levels in monoclonal gammopathies: correlation of bone marrow granular lymphocytes to prognosis. Acta Haematol. 1997; 98:150-154. [PubMed: 9352746]

12. Nielsen H, Nielsen HJ, Tvede N, Klarlund K, Mansa B, Moesgaard F, et al. Immune dysfunction in multiple myeloma. Reduced natural killer cell activity and increased levels of soluble interleukin-2 receptors. APMIS. 1991; 99:340-346. [PubMed: 2036217]

13. Beyer M, Kockanek M, Giese T, Endl E, Weihrauch MR, Knolle PA, et al. In vivo peripheral expansion of naive CD4+CD25high FoxP3+ regulatory T cells in patients with multiple myeloma. Blood. 2006; 107:3940-3949. [PubMed: 16410445]

14. Tinofer I, Marschitz I, Henn T, Egle A, Greil R. Expression of a functional interleukin-15 receptor and autocrine production of interleukin-15 as mechanisms of tumor propagation in multiple myeloma. Blood. 2000; 95:610-618. [PubMed: 10627470]

15. Hsu AK, Quach H, Tai T, Prince HM, Harrison SJ, Trapani JA, Smyth MJ, Neeson P, Ritchie DS. The immunostimulatory effect of lenalidomide on NK-cell function is profoundly inhibited by concurrent dexamethasone therapy. Blood. 2011; 117(5):1605-1613. [PubMed: 20978269]

16. Carter CR, Feyler S, Smalle N, Scott GB, Parrish C, Cullen K, et al. Effect of combined dexamethasone/lenalidomide therapy on NK cell-receptor levels in myeloma patients. Blood. 2011; 118(24):6465-6456. [PubMed: 22161851]

17. Rajkumar SV, Jacobus S, Callander NS, Fonseca R, Vesole DH, Williams ME, et al. Lenalidomide plus high-dose dexamethasone versus lenalidomide plus low-dose dexamethasone as initial therapy for newly diagnosed multiple myeloma: an open-label randomized controlled trial. Lancet Oncol. 2010; 11:29-37. [PubMed: 19853510]

18. Mitsiades CS. How “immunomodulatory” are IMIDs? Blood. 2011; 117(5):1440-1441. [PubMed: 21292783]

19. Romagne F, Andre P, Spee P, Zahn S, Anfossi N, Gauthier L, et al. Preclinical characterization of 1-7F9, a novel anti-KIR receptor therapeutic antibody that augments natural killer-mediated killing of tumor cells. Blood. 2009; 114(13):2667-2677. [PubMed: 19553639]

20. Ruggeri L, Capanni M, Urbani E, Perruccio K, Shlomchik WD, Tosti A, et al. Effectiveness of donor natural killer cell alloreactivity in mismatched hematopoietic transplants. Science. 2002; 295:2097-2100. [PubMed: 11896281]

21. Benson DM Jr, Hofmeister CC, Padmanabhan S, Suvannasankha A, Jagannath S, Abonour R, et al. A phase I trial of the anti-KIR antibody IPH2101 in patients with relapsed/refractory multiple myeloma. Blood. 2012; 120(22):4324-4333. [PubMed: 23033266]

22. Benson DM Jr, Bakan CE, Zhang S, Collins SM, Liang J, Srivastava S, et al. IPH2101, a novel anti-inhibitory KIR antibody, and lendalidomide combine to enhance the natural killer cell versus multiple myeloma effect. Blood. 2011; 118(24):6387-6391. [PubMed: 22031859]

23. Godfrey J, Benson DM Jr. The role of natural killer cells in immunity against multiple myeloma. Leuk Lymphoma. 2012; 53:1666-1676. [PubMed: 22423650]

24. Kroger N, Shaw B, Iacobelli S, Zabelina T, Peggs K, Shimoni A, et al. Comparison between antithymocyte globulin and alemtuzumab and the possible impact of KIR-ligand mismatch after dose-reduced conditioning and unrelated stem cell transplantation in patients with multiple myeloma. Brit J Haematol. 2005; 129:631-643. [PubMed: 15916686]

25. Richardson P, Jagannath S, Hussein M, Berenson J, Singhal S, Irwin D, et al. Safety and efficacy of single-agent lenalidomide in patients with relapsed and refractory multiple myeloma. Blood. 2009; 114:772-778. [PubMed: 19471019]

26. Weber D, Chen C, Niesvizky R, Wang M, Belch A, Stadtmauer EA, et al. Lenalidomide plus dexamethasone for relapsed multiple myeloma in North America. New Eng J Med. 2007; 357:2133-2142. [PubMed: 18032763]

27. Dimopoulos M, Spencer A, Attal M, Prince HM, Harousseau JL, Dmoszynska A, et al. Lenalidomide plus dexamethasone for relapsed or refractory multiple myeloma. New Eng J Med. 2007; 357:2123-2132. [PubMed: 18032762] 
28. Allegra A, Penna G, Alonci A, Russo S, Greve B, Innao V, et al. Monoclonal antibodies: potential new therapeutic treatment against multiple myeloma. Eur J Haematol. 2013; 90(6):441-468. [PubMed: 23506222]

29. Chu J, Deng Y, Benson DM, He S, Hughes T, Zhang J, et al. CS1-specific chimeric antigen receptor (CAR)-engineered natural killer cells enhance in vitro and in vivo anti-tumor activity against human multiple myeloma. Leukemia. 2014; 28(4):917-927. [PubMed: 24067492]

30. Wang L, Jin N, Schmitt A, Greiner J, Malcherek G, Hundemer M, et al. T cell-based targeted immunotherapies for patients with multiple myeloma. Int J Cancer. 2015; 136(8):1751-1768. [PubMed: 25195787]

31. Benson DM, Byrd JC. CS-1 directed monoclonal antibody therapy for multiple myeloma. J Clin Oncol. 2012; 30(16):2013-2015. [PubMed: 22547601]

32. Nijhof IS, Lammerts van Bueren JJ, van Kessel B, Andre P, Morel Y, Lokhorst HM, et al. Daratumumab-mediated lysis of primary multiple myeloma cells is enhanced in combination with the human anti-KIR antibody IPH2102 and lenalidomide. Haematologica. 2015; 100(2):263-268. [PubMed: 25510242]

33. Atanackovic D, Luetkens T, Kroger N. Coinhibitory molecule PD-1 as a potential target for the immunotherapy of multiple myeloma. Leukemia. 2014; 28(5):993-1000. [PubMed: 24153012] 


\section{Statement of Translational Relevance}

In addition to directly inducing apoptosis of multiple myeloma (MM) cells, lenalidomide modulates expansion and activation of natural killer (NK) cells. NK cells appear to play an important role in the immune response to MM; however, MM cells utilize NK cell specific immunoevasive strategies, including expression of inhibitory killer immunoglobulin-like receptor (KIR) ligands that prevent NK cell recognition and lysis. IPH2101, a non-depleting, $\mathrm{IgG}_{4}$ antibody against common inhibitory KIR, was shown to be safe and well tolerated agent in relapsed/refractory MM with evidence of disease stabilization in $34 \%$ of patients as a single agent. The present findings suggest that the use of lenalidomide for NK cell stimulation and IPH2101 to release NK cells from inhibitory signaling is safe, tolerable and associated with signs of clinical efficacy. These results justify further research into combinatory, innate immune therapy for MM devoid of corticosteroid use. 


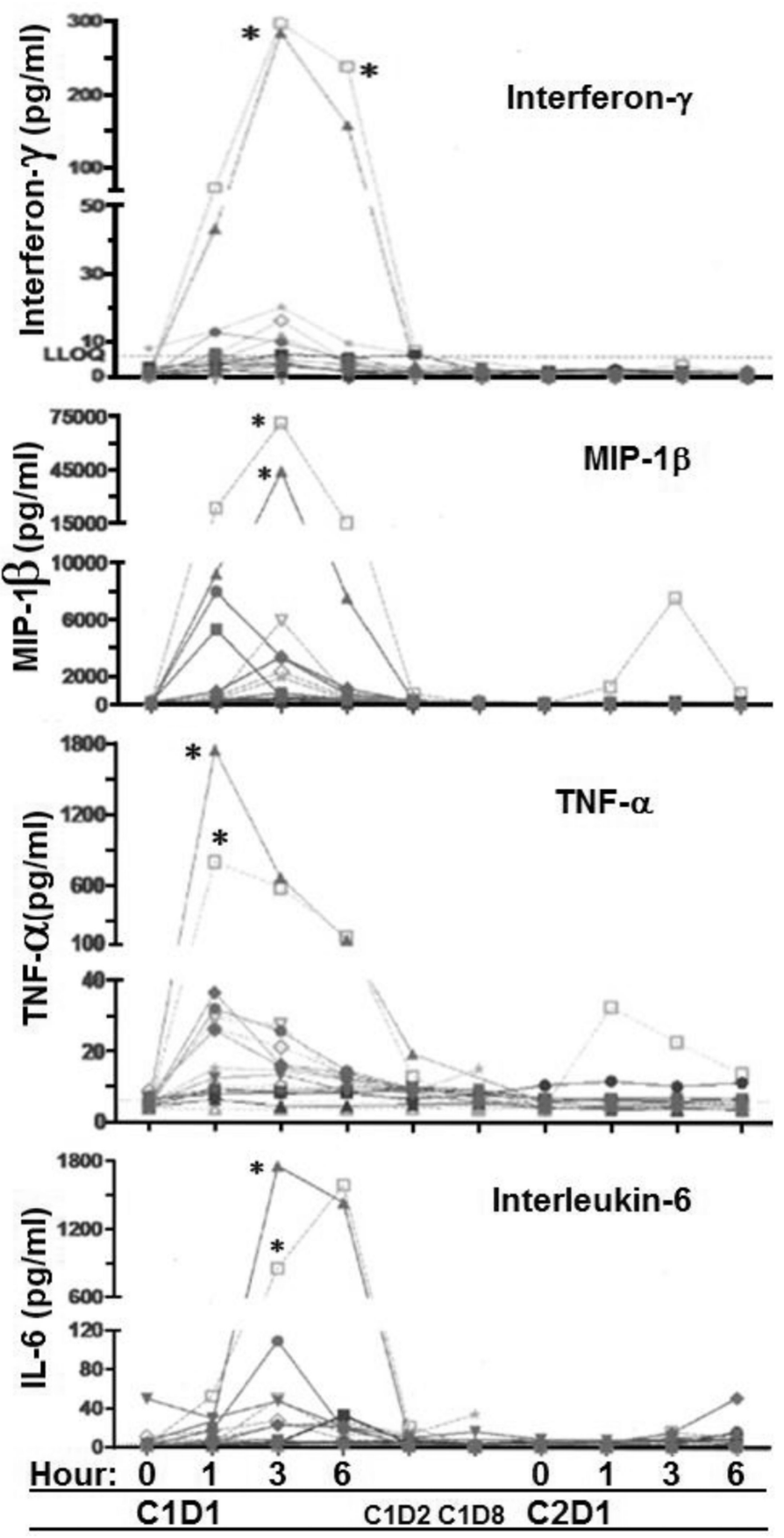

Figure 1.

Changes in pro-inflammatory cytokines observed in cycles 1 and 2 . The 2 patients who experienced a potential DLT of clinically evident, infusion-related, cytokine release syndrome are marked (“*”). 


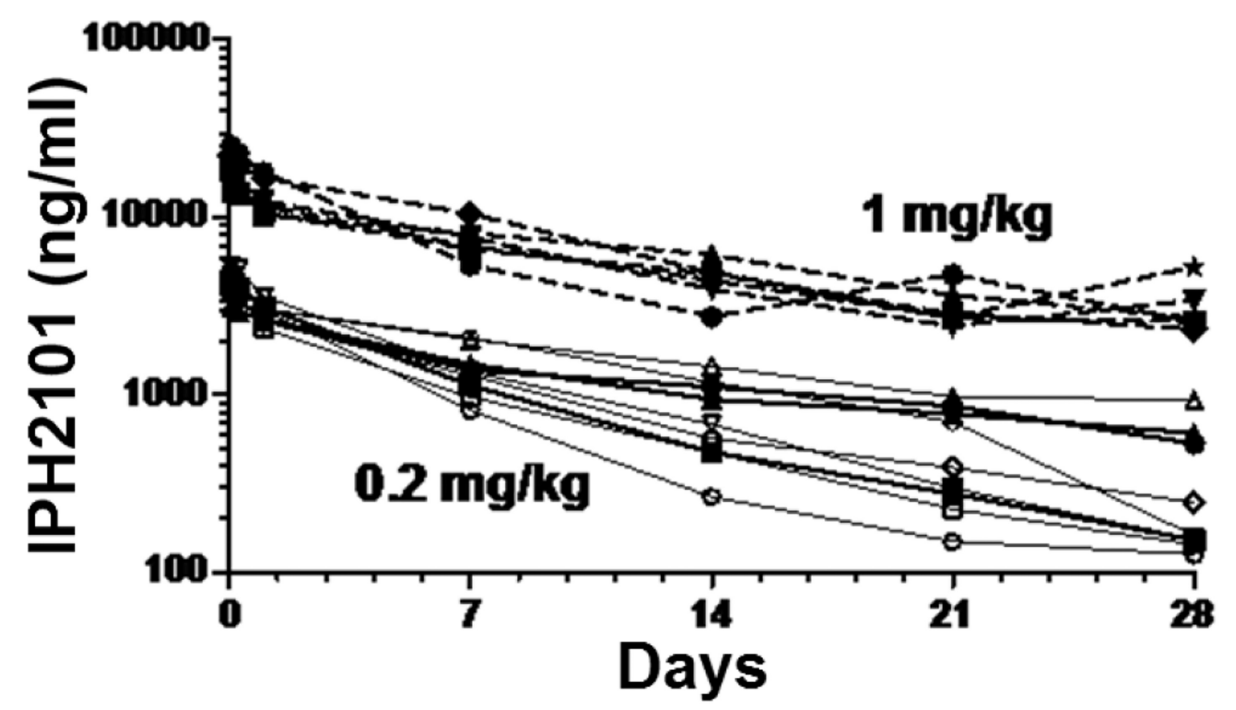

A.

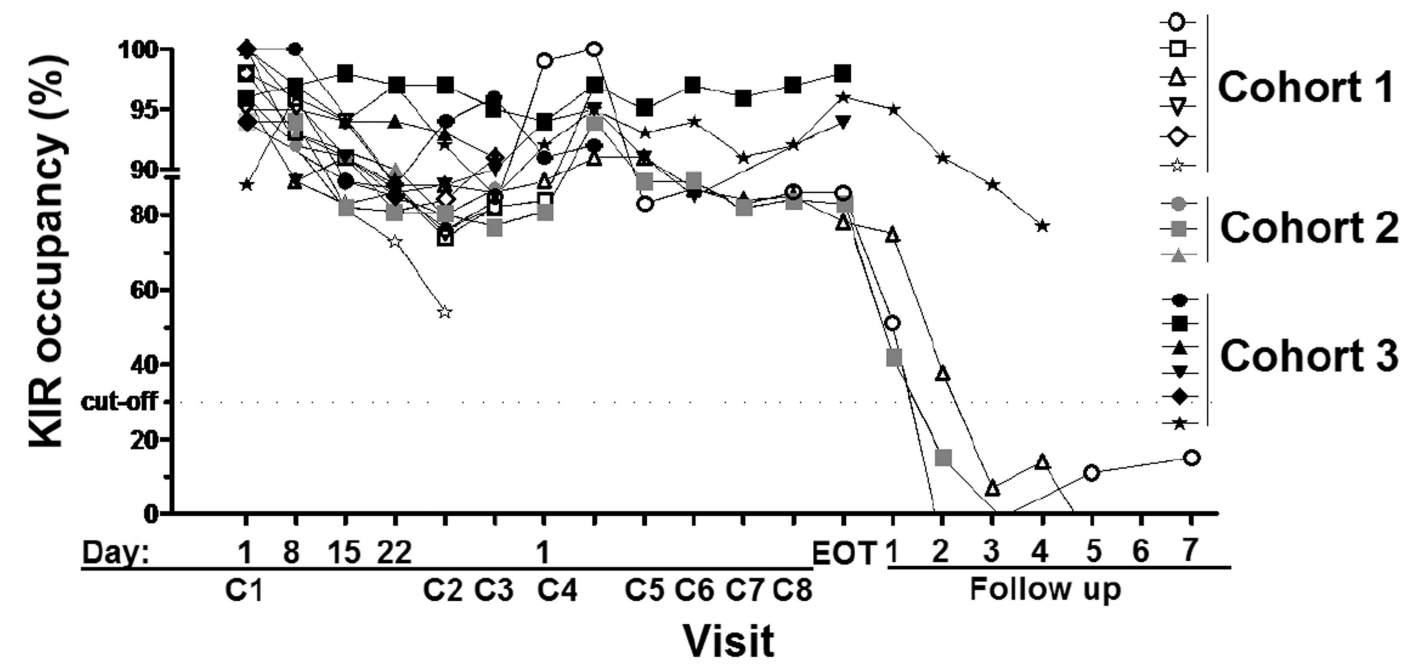

B. 


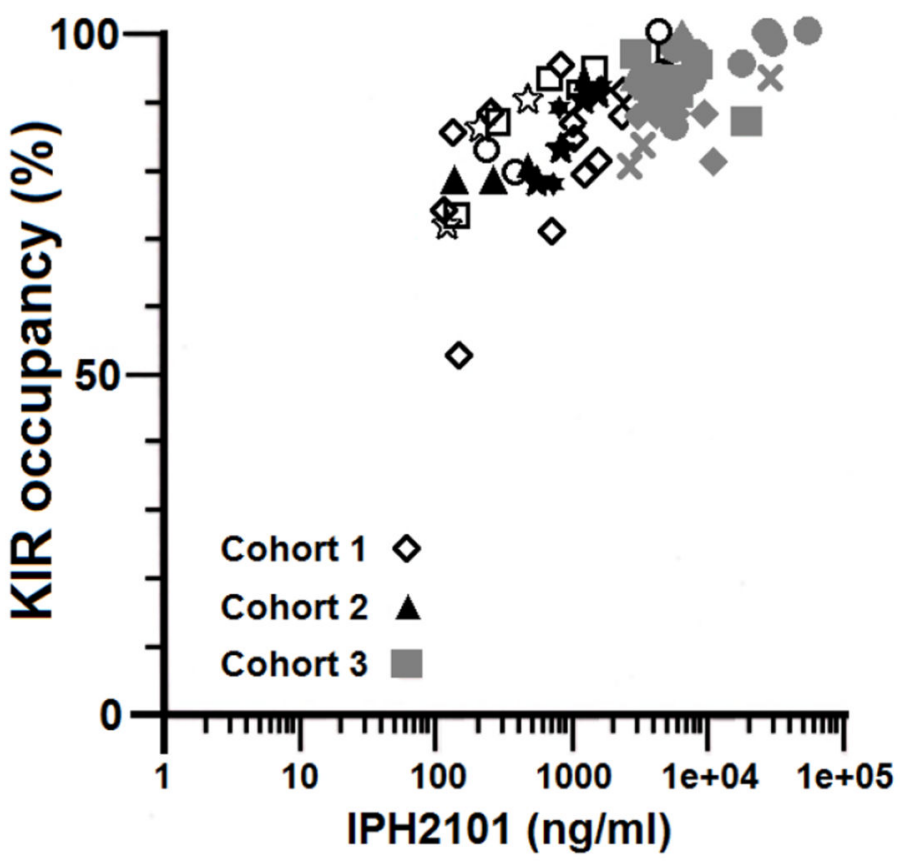

C.

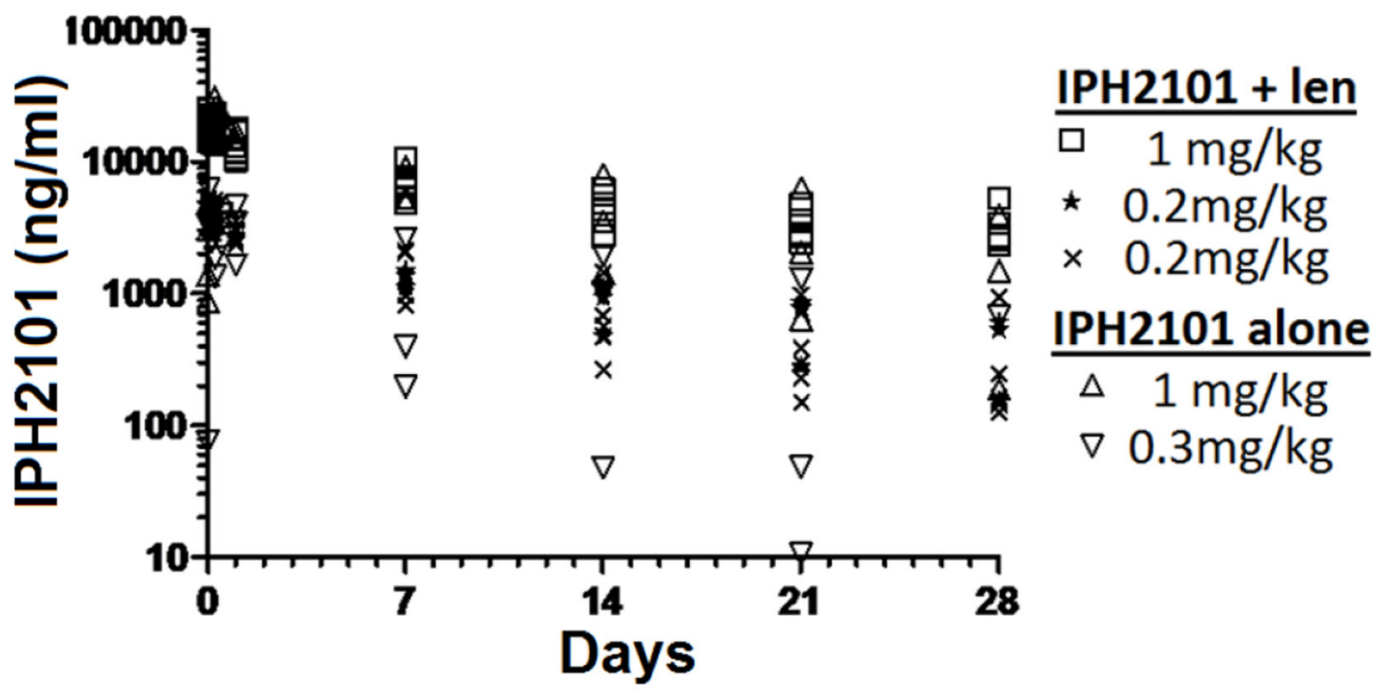

D.

Figure 2.

(A.) Pharmacokinetic data demonstrate the IPH2101 serum concentration over time is associated with dose. (B.) Pharmacodynamic data suggest that KIR occupancy is a function of IPH2101 dose and time. Most patients treated at $1 \mathrm{mg} / \mathrm{kg}$ dosage achieved full KIR occupancy over the dosing interval of 28 days. (C.) IPH2101 dose correlates with KIR occupancy. (D.) Co-administration of lenalidomide with IPH2101 does not appear to affect serum concentration of IPH2101 as compared to data from a prior single-agent IPH2101 study. 

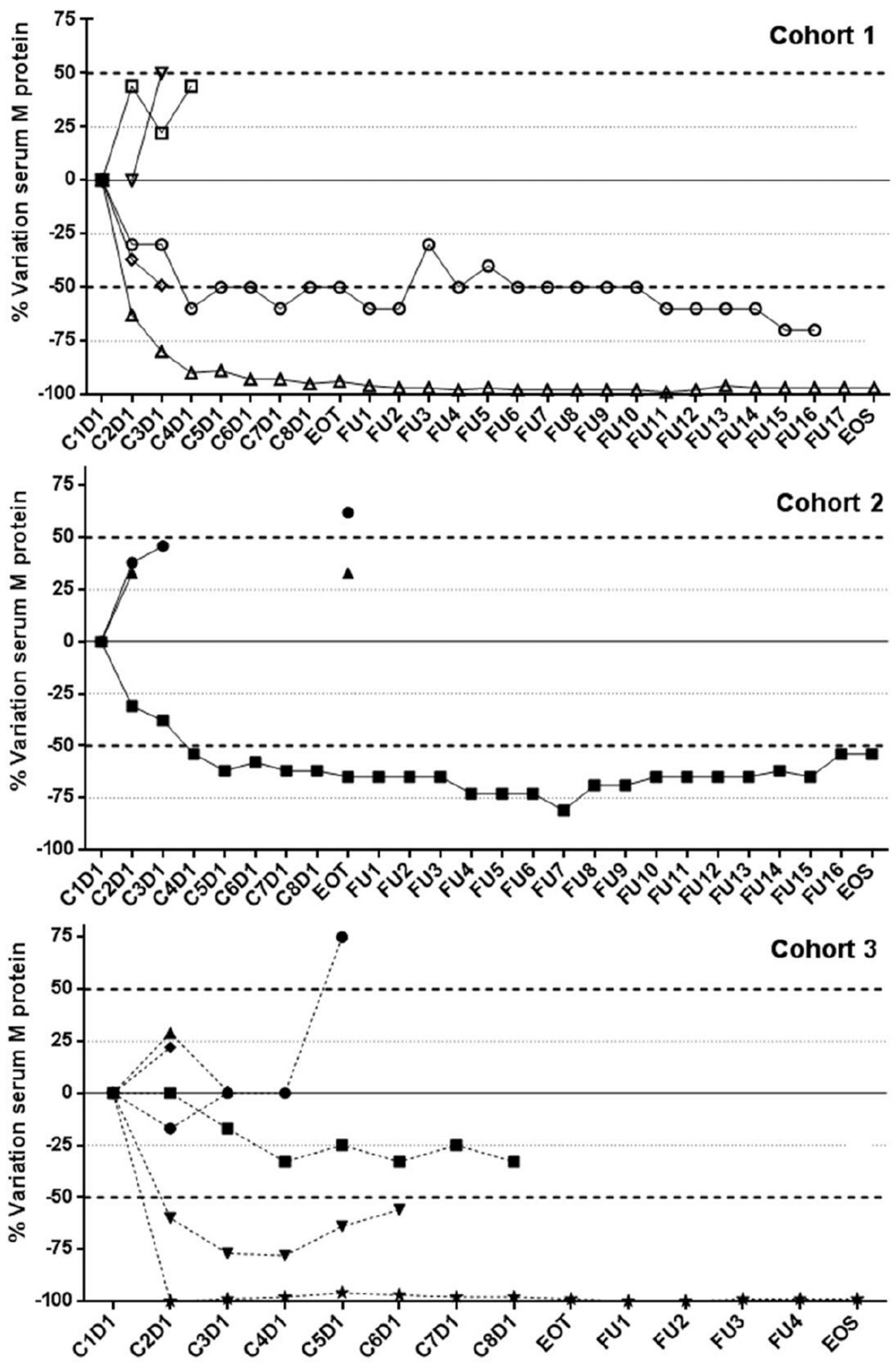

A. 


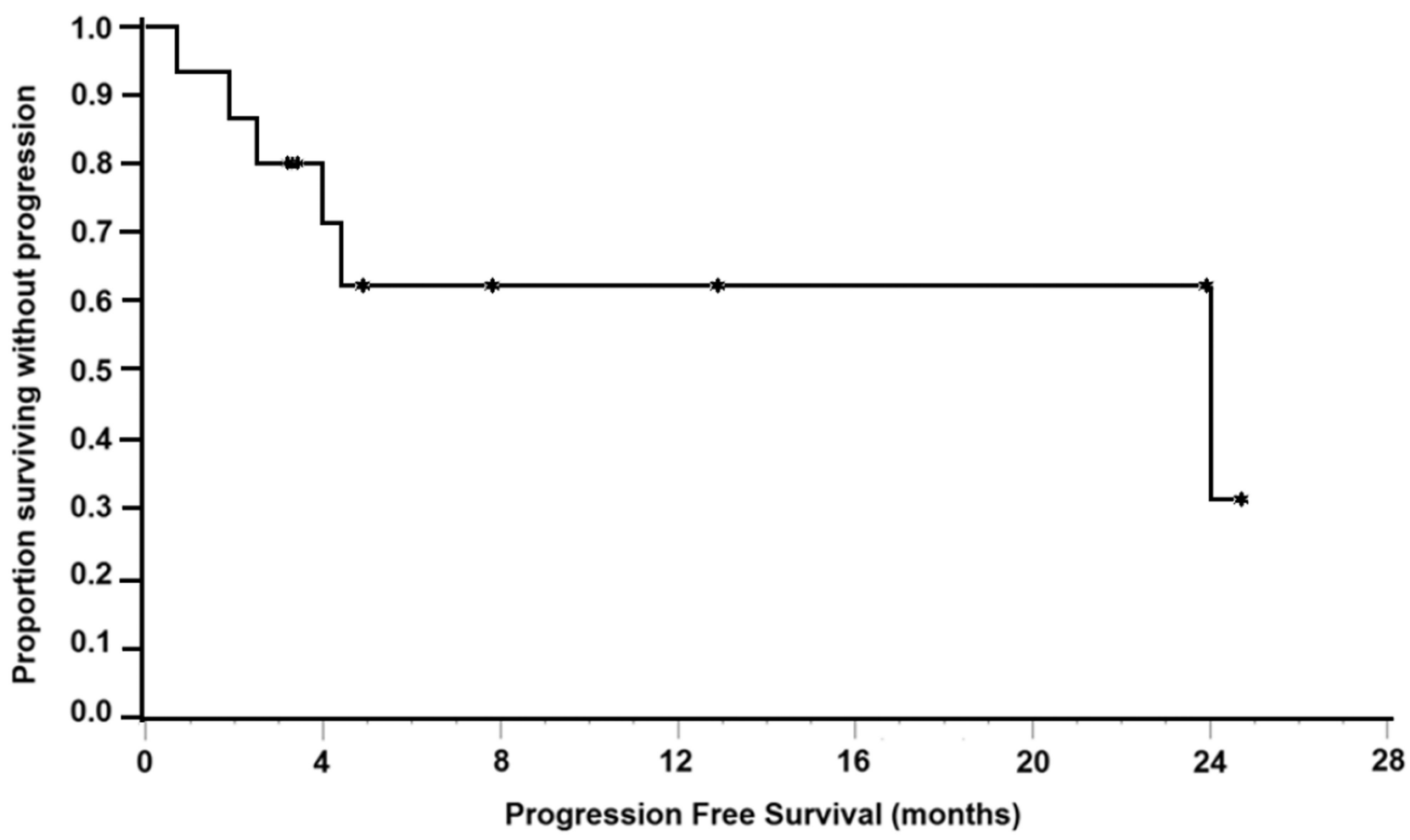

B.

Figure 3.

(A.) Serial changes in individual subject MM biomarkers per cohort. (B.) Progression free survival curve for enrolled subjects in the present trial. 
Table 1

Patients $(n=15)$ demographics, disease, and prior treatment characteristics

\begin{tabular}{lc}
\hline Demographics & Value \\
Sex, female, no. (\%) & $5(33)$ \\
Median age, yr (range) & $60(39-76)$ \\
Prior treatments, no of patients $(\%)$ & \\
Corticosteroids & $11(73)$ \\
Anthracyclines & $5(33)$ \\
Alkylating agents & $12(80)$ \\
Bortezomib & $11(73)$ \\
Lenalidomide & $10(66)$ \\
Thalidomide & $5(33)$ \\
Other & $4(27)$ \\
Median time, diagnosis to enrollment, y (range) & $3.5(1.3-10.1)$ \\
International Staging System stage, no., \% & \\
1 & $8(53)$ \\
2 & $3(20)$ \\
3 & $0(0)$ \\
Unknown & $4(27)$ \\
Type of M-protein, no of patients (\%) & \\
IgG & $6(40)$ \\
IgA & $3(20)$ \\
Light chain & $6(40)$ \\
Sytogenetics (available on n=11), \% & \\
\hline High risk & 55 \\
\hline
\end{tabular}



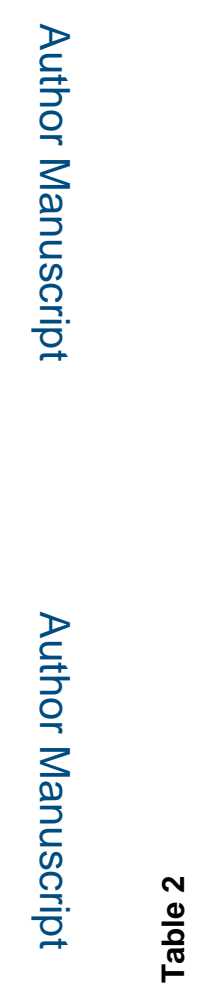

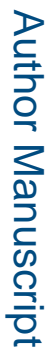

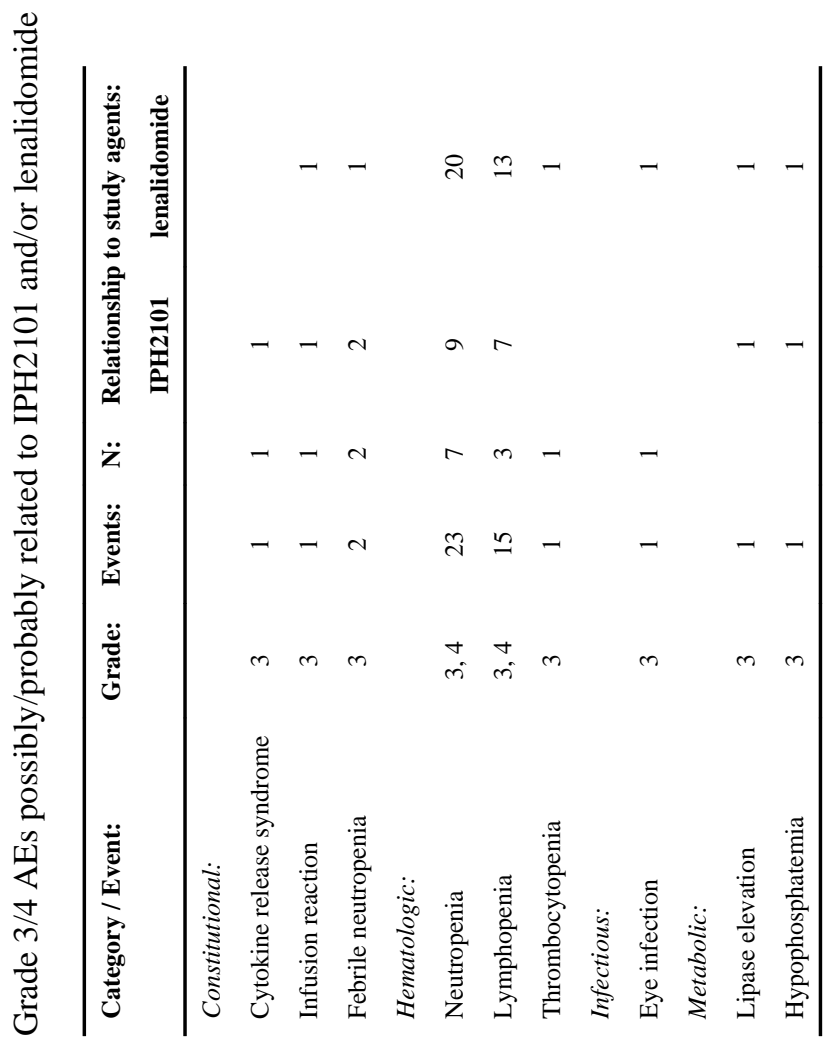

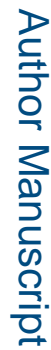

Clin Cancer Res. Author manuscript; available in PMC 2016 September 15. 

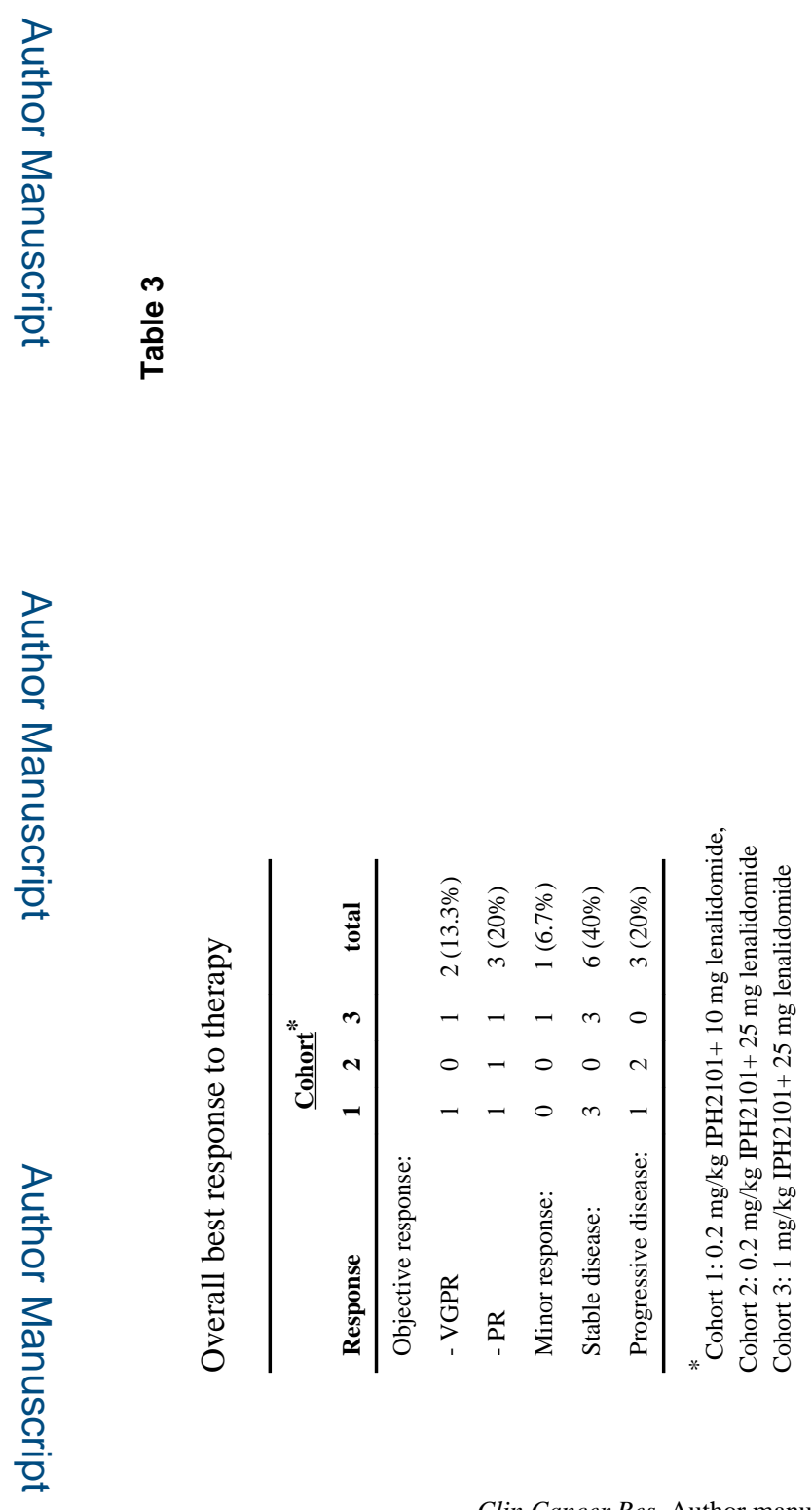

Clin Cancer Res. Author manuscript; available in PMC 2016 September 15. 